\title{
Efecto de la suplementación con ensilaje de estiércol porcino sobre los indicadores productivos en bovinos Hartón del Valle
}

\author{
Effect of supplementation with pig manure silage on the \\ productive indicators bovine Hartón del Valle
}

\section{Efeito da suplementação com silagem de estrume de suínos sobre os indicadores de produção de bovino Hartón del Valle}

\author{
Adrian R. Riascos - Vallejos ${ }^{1}$, José E. Apráez-Guerrero ${ }^{2}$, Diana P. Vargas $M^{3}$, \\ Aldemar Londoño-Arcila ${ }^{4}$ \\ 1 Zoot, Esp, MSc Unidad de ganadería. Centro Agropecuario de Buga, Servicio Nacional de Aprendizaje - SENA, Regional \\ Valle; Guadalajara de Buga, Valle del Cauca, Colombia \\ 2 Zoot, Esp, MSc, PhD, Docente Departamento de Producción y Procesamiento Animal. Universidad de Nariño San Juan de \\ Pasto, Nariño, Colombia. \\ 3 Ing. Agr, MSc, Estudiante de Doctorado en Ingeniería de alimentos. Universidad de Campinas. Sao Paulo, Brasil. \\ 4 Estudiante de Zootecnia, Universidad Nacional de Colombia, Palmira-Colombia. \\ Email: rolando.riascos@gmail.com
}

Recibido: 06 de febrero de $2017 \quad$ Aceptado: 13 de junio de 2018

\begin{abstract}
Resumen
Se evaluó el efecto de la suplementación con ensilaje de estiércol porcino sobre indicadores productivos de novillas raza Hartón del Valle, Se utilizó un diseño Irrestrictamente al azar, se aplicó pruebas de normalidad distributiva, análisis de varianza y prueba de Tukey, los animales fueron asignados al azar a dos grupos experimentales. Los dos grupos consumieron como base forrajera, pasto King grass morado (Pennisetum purpureum CV King grass) y los ensilajes suministrados a las novillas contenían dos niveles de inclusión del estiércol 15\% (T1) y 30\% (T2). Se seleccionaron 12 hembras destetadas de la línea Zanjón Hondo, con peso promedio de $218 \mathrm{~kg} \pm 6 \mathrm{~kg}$, de 12 meses de edad, donde se determinaron por un periodo de 45 días, los indicadores productivos de consumo, cambio de peso y conversión alimenticia. Los dos niveles de suplementación con porquinaza no afectaron (P>0.05) el consumo voluntario de materia seca (CVMS) 6,58 (T1) y 7,06 (T2) kg/MS/animal/día. Sin embargo, si hubo diferencias $(\mathrm{P}<0.05)$ en ganancia diaria de peso, las cuales fueron de 0,53 para T1 y 0,38 kg/día en T2. La conversión alimenticia se estimó en 11,66 en T1 y 18,89 en T2 (P<0.05). Se concluye que los indicadores productivos de novillas Hartón del Valle resultan satisfactorios al incorporar $15 \%$ de estiércol de cerdo deshidratado en el ensilaje como el alimento suplementario en el periodo de levante.
\end{abstract}

Palabras clave: Gliricidia sepium, suplementación, toxicidad, porquinaza, Pennisetum purpureum.

\section{Abstract}

The effect of porcine manure silage supplementation on productive indicators of Hartón del Valle heifers was evaluated. An Irresistibly Random design was used, distributive normality tests, analysis of variance and Tukey test were applied. The 
animals were randomly assigned to two experimental groups. Both groups consumed forage purple king grass (Pennisetum purpureum CV King grass) and silage supplied to heifers contained two levels of inclusion of manure 15\% (T1) and 30\% (T2). Twelve weaned females were selected from the Zanjón Hondo line, with a mean weight of $218 \mathrm{~kg} \pm 6 \mathrm{~kg}$, of 12 months of age, where were determined for a period of 45 days, productive indicators of consumption, weight change and feed conversion. The two levels of supplementation with porkase did not affect $(P>0.05)$ voluntary dry matter intake $(C V M S)$ $6.58(\mathrm{~T} 1)$ and $7.06(\mathrm{~T} 2) \mathrm{kg} / \mathrm{d}$.m./animal/día. However, there were differences $(\mathrm{P}<0.05)$ in daily weight gain, which were 0.53 for T1 and $0.38 \mathrm{~kg} /$ day for T2. Feed conversion was estimated at 11.66 for T1 and 18.89 for $\mathrm{T} 2$ ( $\mathrm{P}<0.05)$. It is concluded that the productive indicators of Hartón del Valle heifers are satisfactory by incorporating 15\% of dehydrated pig manure in silage as the supplementary feed in the period of rising.

Key words: Gliricidia sepium, supplementation, toxicity, porquinaza, Pennisetum purpureum.

\section{Resumo}

Avaliou-se o efeito da suplementação com silagem de suínos em indicadores produtivos de novilhas Hartón del Valle, utilizando-se um desenho Irresistivelmente Random, testes de normalidade distributiva, análise de variância e teste de Tukey, os animais foram divididos aleatoriamente em dois grupos experimentais. Ambos grupos consumiram como base de forragem a grama king grass roxa (Pennisetum purpureum CV King grass) e os silagem para as novilhas tinham dois níveis de inclusão de estrume de 15\% (T1) e 30\% (T2). Foram selecionados 12 fêmeas recém-desmamados da linha Zanjón Hondo, com um peso médio de $218 \mathrm{~kg} \pm 6 \mathrm{~kg}, 12$ meses de idade, onde foram determinadas por um período de 45 dias os indicadores de consumo produtivas, mudança de peso e conversão alimentar. Os dois níveis de suplementação porquinasa não afetou (P>0.05) a ingestão voluntária de matéria seca (CVMS) 6,58 (T1) e 7,06 (T2) kg/MS/animal/día. No entanto, se houve diferenças $(\mathrm{P}<0.05)$ no ganho de peso diário, que foram 0,53 para T1 e $0,38 \mathrm{~kg} /$ dia para T2. A conveção alimentar estimo-se em 11,66 em T1 e 18,89 em T2 ( $<<0.05)$. Conclui-se que os indicadores de novilhas produtivas Harton del Valle são satisfatórios para incorporar $15 \%$ da silagem de estrume de porco deidratado como o alimento-suplemento no período de criação.

Palavras-chave: Gliricidia sepium, suplementação, toxicidade, porquinaza, Pennisetum purpureum.

\section{Introduccion}

El actual cambio climático ha dejado notables catástrofes en el sector pecuario, una de estas es el Fenómeno del Niño, una amenaza acompañada de fuertes sequías las cuales terminan con un desabastecimiento del alimento para los animales; cuando esto ocurre se hace necesario encontrar maneras de suplementación animal (González et al., 2010), la tarea más difícil es encontrar alimentos que proporcionen nutrientes para satisfacer los requerimientos del animal, pero que a su vez no sean de gran valor económico o compitan con la alimentación humana; (Pérez, Heredia, y Flores, 2002) Actualmente la disposición de la materia fecal proveniente de los cerdos, se ha convertido en un asunto de impacto ecológico, pues además de generar volúmenes altos causan toxicidad al suelo. La manera más fácil y económica de manejar eficientemente estos residuos, es su utilización en la alimentación bovina, con más del 22\% de proteína cruda (PC). (Duarte Vera, Magaña Caballero, y Rodriguez Garza, 1990).

En climas tropicales, donde existe producción de cerdos, la reutilización de la cerdaza en la alimentación de rumiantes se considera una alternativa de baja inversión tanto para el ganadero como para el porcicultor, produciendo un ingreso adicional a la actividad porcina y constituye una gran ayuda en el engorde de ru- miantes (Heredia, 2012). Su composición cuenta con un alto contenido de materia orgánica, (Hochstrasser, Salazar, Dominguez, Dela-Mora, Galindo, Xelhuantzi y Perez, 2012) ácidos grasos volátiles, nitrógeno, fósforo, potasio y otros minerales (Garcia, 2000) por estas razones, el ensilaje de cerdaza es uno de los procesos que presenta mayores número de ventajas para la reutilización de los nutrimentos encontrados en las heces sólidas de cerdos; transforma los olores desagradables, conserva y modifica de manera positiva los nutrimentos presentes en las excretas, además de reducir el riesgo de transmisión de microorganismos patógenos, tanto para animales y humanos. (González, Venegas, Orozco, Martínez, García, Ramos, y Rodríguez, 2010).

Estudios ratifican que después de 30 días de fermentación, el ensilaje elimina la presencia de los cinco virus analizados PRRS, PVP, PCV, circovirus tipo I y II y HVP-I (PCV-I y PCV-II), y el herpesvirus porcino tipo I (HVP-I). Parásitos, larvas de nematodos (Guillermin, 1995) Salmonella spp. El estiércol de cerdo fue tres veces menos tóxico que el estiércol de aves (Gupta, 1990). Se encontró inocuo para Coliformes totales y fecales. Desde el sexto día de fermentación, las bacterias del género Clostridium, de tipo sulfito reductores, son totalmente eliminadas del ensilaje. Para Staphylococcus, de tipo coagulasa positivo, se indican valores compatibles con 
la salud animal y humana, y por tanto, es posible su incorporación hasta el $40 \%$ de porcinaza. (Estrada y Aranda, 2011). Por tanto, puede ser utilizado en la alimentación animal sin consecuencias en la productividad y la calidad del producto (Castellanos, Salazar, Hernandez, Domingez Araujo, y Barrera, 2013); sin ser difícil colectarla, pues se considera que cada cerdo excreta entre 0,5 y 0,8 kg de heces y 2,1 kg de orina por cada $100 \mathrm{~kg}$ de peso (G. Flachowsky, 1997).

El rumiante tiene la capacidad de utilizar alimentos de bajo valor nutritivo y convertirlos en carne, gracias a la acción enzimática de los microorganismos del rumen, esto hace posible que los rumiantes obtengan energía de los polisacáridos que no pueden ser utilizados eficientemente por monogástricos. Además, los microorganismos ruminales pueden sintetizar proteína a partir del nitrógeno no proteico y convertirlo fácilmente en carne y leche para consumo humano. De esta manera los antecedentes del uso de excertas de cerdos en la suplementación de rumiantes, suministrada tanto fresca como deshidratada, o en ensilaje (Garcia, 2000). No obstante, es aconsejable utilizar subproductos fibrosos para disminuir la humedad y el contenido de amonio (Pérez, Heredia, y Flores, 2002). La proporción de la cerdaza: material fibroso varía de 20:80 hasta 25:75 cuando se utiliza toda la planta (Sutton, 1988). Una alternativa que enriquece la dieta es el de pasto guinea (Panicum maximum) y el matarraton (Gliricidia sepium) una leguminosa que predomina en el trópico, y que a su vez ofrece grandes rendimientos como suplemento en bovinos, ya que no solo aporta hasta el $20 \%$ de PC, (Lemos, 2014) protegida por compuestos fenólicos en las hojas que le proveen cierta capacidad de proteína sobrepasante, sin que exista reportes de toxicidad aún en animales alimentados en su totalidad a base de esta planta (Araque, Quijada, D’Aubeterre, Páez, Sánchez, y Espinoza, 2006) y altos contenidos de macro minerales (Vollink, 1993), bajo contenido de taninos 40g/kg PV (Cecconello, 2003), efectúa fijación de nitrógeno atmosférico al suelo, útil en sistemas silvopastoriles, barreras cortaviento, cercas vivas y bancos de proteína.

Aunque algunos consideran factores anti nutricionales en el ensilaje; bajas concentraciones de taninos pueden incrementar el nivel de aminoácidos azufrados que entran al torrente sanguíneo, niveles altos de taninos mezclados con especies ricas en nitrógeno soluble mejoran la degradación proteica y la absorción del nitrógeno disminuyendo el efecto de los compuestos tóxicos (Bhat, 1998); En la presente investigación se evaluó el comportamiento productivo de terneros de la raza hartón del valle alimentados con dietas en las que se incluyó ensilaje de estiércol de cerdo y una mezcla forrajera de matarratón y King grass ( $P$ ennisetum purpureum $\mathrm{CV}$ ). (Simon, 2003).

\section{Materiales y métodos}

El estudio se realizó en el Centro Agropecuario de Buga, SENA, ubicado en el municipio de Guadalajara de Buga, departamento del valle del cauca, colombiano, a una altitud de $950 \mathrm{msnm}$, zona de vida de Bosque Seco Tropical (bs-T) según la clasificación de Holdrige (Espinal, 1968) con una temperatura promedio de $25^{\circ} \mathrm{C}$.

Se seleccionaron 12 hembras destetadas de la raza hartón del valle de la línea Zanjón hondo, con un peso promedio de $218 \mathrm{~kg} \pm 6 \mathrm{~kg}$ y 12 meses de edad con los que se formaron dos grupos homogéneos de seis novillas por grupo. Se evaluaron dos niveles de inclusión del estiércol, $15 \%$ y $30 \%$ en el ensilaje fresco que se suministró como suplemento de acuerdo a la composición bromatológica (Tabla 1), los dos grupos consumieron King grass morado (Pennisetum purpureum CV King grass), fue cortado a una edad de 70 días, picado diariamente pesado y suministrado fresco el mismo día, pesando el rechazo para calcular el consumo, de acuerdo las tablas de Nutrient Requierements of Dairy Cattle: Seventh Revised Edition, (2001). El ensilaje elaborado se suministró a razón de un 30 $\%$ del consumo total de materia seca, por un periodo de 45 días. Los animales se sometieron a un periodo de adaptación de 15 días, los cuales se pesaron de manera individual al inicio del experimento y posteriormente cada 15 días, previo ayuno de $12 \mathrm{~h}$. Cada grupo se alojarón en un corral en el que se dispuso de un comedero lineal de plástico y agua limpia a voluntad los cuales estuvieron estabulados durante todo el periodo experimental.

Para la preparación del ensilaje semisólido, se hizo la recolección del estiércol de la unidad de cría de la granja porcicola del Centro Agropecuario de Buga; se deshidrato bajo techo por dos días, se mezcló con los demás ingredientes y se preparó en un contenedor de plástico de 1000 litros, el tiempo de fermentación fue de 30 días. Para la mezcla del ensilaje se tuvo en cuenta los análisis proximales realizados en el laboratorio de análisis de control de calidad de alimentos del Centro Agropecuario de Buga.

Consumo de alimento: La cantidad de suplemento ofrecido a cada animal, fue de 33,4 \% kg de MS en función del consumo total el cual se pesó diariamente y se ofreció en comederos individuales. Después 
Tabla 1. Análisis proximal de las materias primas y tratamientos utilizados

\begin{tabular}{|c|c|c|c|c|c|c|c|}
\hline \multirow{2}{*}{ Materias Primas } & \multicolumn{2}{|c|}{ Trat. (\%) } & \multicolumn{5}{|c|}{ Nutrientes } \\
\hline & T1 & T2 & MS & PC & EE & FC & Cenizas \\
\hline Estiércol de cerdo & 30 & 15 & 30,21 & 17,21 & 5,21 & 15,6 & 12,96 \\
\hline Matarratón & 10 & 15 & 28,1 & 27,5 & 3,2 & 25,2 & 16,24 \\
\hline Pasto Guinea & 12 & 12 & 29,21 & 8,5 & 0,68 & 39,21 & 14,34 \\
\hline Maíz molido & 23 & 23 & 86,82 & 8,15 & 4,03 & 2,4 & 1,34 \\
\hline Mogolla & 1,5 & 6 & 59,6 & 12,14 & 5,12 & 10,21 & 5,41 \\
\hline Urea & 0,5 & 1 & 98 & 273 & 0,1 & 0 & 0,7 \\
\hline Aceite vegetal & 2 & 2 & 81,2 & 0 & 100 & 0 & 0 \\
\hline Melaza & 10 & 10 & 74,7 & 3,7 & 0,09 & 0 & 10,1 \\
\hline Sal mineralizada & 1 & 1 & 0 & 0 & 0 & 0 & 97 \\
\hline Ensilaje & \multicolumn{2}{|c|}{ Tratamiento 1} & 51,80 & 14,2 & 5,1 & 13,91 & 10,64 \\
\hline Ensilaje & \multicolumn{2}{|c|}{ Tratamiento 2} & 53,24 & 14,48 & 5,22 & 15,28 & 10,45 \\
\hline Pasto King grass & \multicolumn{2}{|c|}{ Base forrajera } & 14,72 & 8,29 & 2,1 & 15,6 & 12,45 \\
\hline
\end{tabular}

MS: materia seca, PC: proteína cruda, EE: extracto etéreo, FC: fibra cruda.

de 24 horas del suministro el suplemento rechazado fue pesado, para determinar el consumo efectivo de suplemento, se determinó restándole a la cantidad de suplemento ofrecido menos la cantidad rechazada del mismo día; el resultado se expresó en kg.

El pasto King grass como base forrajera, se suministró en relación a su consumo total de su peso vivo, se determinó el consumo promedio mediante la relación de la cantidad ofrecida menos la cantidad rechazada.

Ganancia diaria de peso: Para obtener los cambios de peso vivo, los animales se pesaron individualmente al inicio y posteriormente cada 15 días. Al peso registrado al final, se le restó, el peso registrado al inicialmente y se dividió sobre el número de días del periodo experimental.

Conversión alimenticia aparente: Se determinó mediante la relación del suplemento consumido y el incremento de peso que se obtuvo durante el periodo experimental.

\section{Análisis Estadístico}

Se utilizó un diseño Irrestrictamente al azar, se aplicó Los parámetros estadísticos de normalidad distributiva, valores promedio $(\mathrm{X})$, desviación estándar (DE), coeficiente de variación (CV) para cada variable. Las comparaciones entre grupos se realizaron mediante análisis de varianza y una prueba de Tukey para la diferencia entre medias, con un nivel de significancia del 95\%. Los cálculos se realizaron con el paquete estadístico Minitab vs 16.2.4.4.

\section{Resultados}

\section{Consumo de alimento}

Para la variable de consumo de alimento, los valores promedio al final del periodo para cada tratamiento fueron: T1: 6,58 y T2: $7,06 \mathrm{~kg} / \mathrm{MS} /$ animal/dia se puede inferir que no se presentaron diferencias estadísticas entre los dos tratamientos ( $P>0,05)$. (Tabla 2).

Al respecto cuando se utilizan materiales fibrosos de baja cantidad de carbohidratos fermentables, los niveles de cerdaza varían de 10 al 30\% y los de material forrajeros de 40 a 60\% (Ramirez, 1990), todo ello con el fin de no disminuir la palatabilidad; pues aunque existen investigaciones realizadas con más del 60\% de inclusión en estiércol el uso de más del $40 \%$ de estiércol en el ensilaje disminuye la palatabilidad y aumenta el periodo de adaptación (Galindo, et al., 2013). Sin embargo, hay reportes de consumo de 14,1 kg/animal/ día hechos por Rojas y Ojeda, (2002) incorporándose la cerdaza en niveles de 10, 20 y 30\% en sustitución del afrechillo de trigo. Lo cual es mayor a lo reportado en esta investigación para los dos tratamientos.

\section{Ganancia diaria de peso y conversión alimenticia aparente:}

Los valores promedio al final del periodo, para cada tratamiento fueron: T1: 0,530 kg y T2: 0,380 kg. En cuanto a la ganancia diaria de peso, se puede decir que presentaron diferencias estadísticas entre los dos 
Tabla 2. Consumo de materia seca de forraje y ensilaje durante el período experimental ( $\mathrm{kg} / \mathrm{animal} / \mathrm{día})$

\begin{tabular}{|c|c|c|c|c|}
\hline \multirow{2}{*}{ Semanas } & \multicolumn{2}{|c|}{ Tratamiento 1 } & \multicolumn{2}{c|}{ Tratamiento 2 } \\
\hline $\begin{array}{c}\text { Consumo } \\
(\mathbf{K g}) \\
\mathbf{M S}\end{array}$ & $\begin{array}{c}\text { Consumo } \\
\mathbf{( K g )} \\
\text { Ensilaje }\end{array}$ & $\begin{array}{c}\text { Consumo } \\
\mathbf{( K g )} \\
\mathbf{M S}\end{array}$ & $\begin{array}{c}\text { Consumo } \\
\mathbf{( K g )} \\
\text { Ensilaje }\end{array}$ \\
\hline 1 & 4,78 & 1,63 & 5,03 & 1,09 \\
\hline 2 & 4,72 & 1,87 & 5,11 & 1,79 \\
\hline 3 & 4,44 & 1,95 & 4,76 & 1,89 \\
\hline 4 & 4,78 & 2,07 & 5,35 & 2,15 \\
\hline 5 & 4,65 & 2,03 & 4,88 & 2,11 \\
\hline 6 & 5,02 & 2,20 & 5,36 & 2,28 \\
\hline 7 & 4,92 & 2,23 & 5,35 & 2,34 \\
\hline Consumo promedio & \multicolumn{2}{|r|}{6,58} & & 7,06 \\
\hline
\end{tabular}

MS: materia seca.

Tabla 3. Resultados indicadores productivos por tratamientos.

\begin{tabular}{|c|c|c|c|}
\hline Tratamiento & $\begin{array}{c}\text { Consumo forraje }+ \text { ensilaje } \\
(\mathrm{Kg})\end{array}$ & GDP (kg/día) & Conversión alimenticia \\
\hline & Medias DE CV & Medias DE CV & Medias DE CV \\
\hline T1 & $6,57^{\mathrm{ns}} \quad 0,88 \quad 10,42$ & $0,56^{\mathrm{a}} \quad 0,054 \quad 9,53$ & $11,66^{\mathrm{a}} 1,14 \quad 9,78$ \\
\hline T2 & $7,06^{\mathrm{ns}} \quad 0,88 \quad 2,48$ & $0,38^{b} \quad 0,058 \quad 15,3$ & $18,89^{b} 6,62 \quad 19,16$ \\
\hline
\end{tabular}

\footnotetext{
ab Medias en la misma columna con distinta letra difieren significativamente $(p<0,05)$

ns No significativo estadísticamente

DE: Desviación estándar

GDP: Ganancia Diaria de Peso
}

tratamientos $(\mathrm{P}<0.05),($ Tabla 3$)$. Los resultados son menores para los dos tratamientos que los encontrados por Garcia Rodirguez (2000) con ganancias de 1,1 kg de novillos en finalización alimentados con una mezcla de estiércol de cerdo deshidratado y rastrojo en una proporción de 3:1. De manera similar se encontraron ganancias de 0,72 a 1,16 Kg/día en toretes cebú y Holstein en crecimiento y finalización (CastriIlón Quintana, Jiménez Pérez, y Bedoya Mejía, 2005).

En cuanto a la conversión alimenticia, los valores promedio al final del periodo, para cada tratamiento fueron: T1: 11,66 y T2: 18,89. En cuanto a la conversión alimenticia aparente se presentaron diferencias estadísticas entre los dos tratamientos $(\mathrm{P}<0.05)$ (Tabla 3).

\section{Discusión}

\section{Consumo de alimento}

Los rumiantes han desarrollado un mecanismo natural para la digestión del alimento que incluye: ácidos grasos volátiles, anaerobiosis, temperatura, presión osmótica y ácidos grasos saturados del rumen; además de enzimas proteolíticas y $\mathrm{pH}$ abomasal que permiten probablemente la eliminación de las bacterias patógenas en el ensilaje consumido. Hay estudios que un consumo 6,72 kg de MS; del ensilado de cerdaza puede ser utilizado en la dieta de vacas en lactación sin desmedro en la producción y cantidad de grasa en leche ni efectos negativos sobre las características fisicoquímicas de la leche y efectos adversos en la sa- 
lud de los animales. (Dominguez, y otros, 2013) Los resultados obtenidos en esta investigación indican que se conoce la capacidad del rumiante para convertir nutrientes de baja calidad entre ellos, las proteínas, las que hidroliza y convierte en nuevas proteínas a partir de nitrógeno no proteico. Estos compuestos tienen diferentes tasas de degradación que probablemente influyen en el comportamiento de los bovinos; esto se debe a que ellas poseen distinta solubilidad y tiempo de permanencia en el rumen, efectos que son condicionados por su diferente susceptibilidad al ataque enzimático de la flora ruminal.

\section{Ganancia diaria de peso}

De acuerdo a lo encontrado en este estudio, trabajos de Alvarez y Gutiérrez, (2011); Ninabanda, (2012). El estiércol fresco de cerdo (30\% BS), melaza de caña (35\% BS) y dos fuentes de fibra (35\% BS): M: planta de maíz (Zea mays) madura, seca y sin mazorca (cañuela, paja, rastrojo o residuos de la cosecha del maíz) y planta de sorgo (Sorghum vulgare) madura y seca sin panoja (paja, rastrojo o residuo de la cosecha de sorgo). En animales encastados de Cebú y Suizo con incremento de peso de 0.945 y $0.922 \mathrm{~kg} /$ día. Es posible que el nitrógeno proveniente del estiercol es utilizado por los microbios del rumen, con lo que aumenta la síntesis de biomasa microbiana, mejoran la degradación de las paredes celulares de los forrajes de baja calidad, (Flachowsky S, 1990; Mancini, 1997) además de la obtención de energía y de aminoácidos útiles para el animal hospedero (Trinci, 1994). Y por debajo a los reportados por Quiñonez (2012), que evaluó una dieta diaria a base de forrajeras matarratón (Gliricidia sepium) en terneros pos destete de ambos sexos quien menciona ganancias de 0,86 kg/día y a ensayos realizados en México con rumiantes alimentados con estiércol fresco (25 al 55\% en base seca), melaza y rastrojo, donde tuvieron incrementos de peso vivo de 0,94 kg/día en toretes cebú y Holstein en crecimiento y finalización (Castrillón Quintana, Jiménez Pérez, y Bedoya Mejía, 2005). Y Urdaneta, (2004) con incrementos en peso de $0,64 \mathrm{~kg} /$ día al suplementar terneras mestizas doble propósito con $6 \mathrm{~kg}$ de caña limpia repicada $+6 \mathrm{~kg}$ de follaje de Gliricidia sepium + pastoreo. Sin embargo los incrementos tan bajos son resultado probable dela genética de la razas criollas compensadas por la adaptabilidad al ambiente.

\section{Conversión alimenticia}

Los resultados encontrados son mayores en T2 y T1 que las encontradas por Pérez, Heredia, y Flores, (2002) que obtuvo resultados en toretes con una dieta integra de excretas frescas, con el $45 \%$ de excretas frescas una conversión de 8.45 ( $\mathrm{kg}$ MS/ $/ \mathrm{kg}$ ganancia de PV) y con 6.52 (Heredia Cruz, 2012) En terneras alimentadas con el $15 \%$ de Concentrado Convencional (CC) y 2) Concentrado con Ensilado de Cerdaza (CE $25 \%$ de inclusión). Sin embargo, son superiores para el tratamiento 1, que los reportados por Rojas y Ojeda, (2002) incorporándose la cerdaza en niveles de 10, 20 y $30 \%$ en sustitución del afrechillo de trigo. Los valores promedio y conversión de alimento de 14,2 kg alimento/kg PV.

Para finalizar el uso de excretas porcinas, tratadas o no por diferentes vías, como material para reciclar dentro de la misma especie, como alimento, no parece ofrecer resultados alentadores, tanto desde el punto de vista de su valor nutritivo, como de los rasgos de comportamiento que determina, a menos que sea sometida a un procedimiento de ensilado con materiales tales como granos o forraje, en dependencia del tipo de animal que va a ser alimentado con este material. Esto de hecho mejoraría el valor nutritivo de las excretas, y extendería el volumen de otros alimentos a suministrar a los animales, a costa de disminuir su valor intrínseco como alimento (Estrada, 2011). Restaría por hacer evaluaciones de tipo económico para determinar la viabilidad del sistema (Ly, 2008)

\section{Conclusiones}

Los indicadores productivos de novillas Hartón del Valle resultan satisfactorios al incorporar $15 \%$ de estiércol de cerdo deshidratado en el ensilaje como el alimento suplementario en el periodo de levante.

La inclusión de estiércol de cerdo en la preparación de ensilajes para la alimentación bovina constituye una alternativa viable para suplementación en épocas de escases de recursos forrajeros.

\section{Agradecimientos}

Agradecimientos los trabajadores de campo de la unidad de ganadería Norbery Gutiérrez Florez, Luz Mary Ortiz y Mauricio Medina aprendiz líder.

\section{Bibliografía}

Alvarez SP, Ernestina-Gutiérrez V. 2011. Engorda de toretes a base de estiércol fresco de cerdo y dos fuentes de fibra en una empresa comercial. Universidad Michoacana de San Nicolás de Hidalgo (UMSNH). Pp. 1-8.

Arango. 1994. El matarratón - Leguminosa forrajera arbórea estratégica en los programas de alimentación de ganaderías tropicales Colombianas. Corporación Colombiana de Investigación Agropecuaria. 
Araque C, Quijada T, D’Aubeterre R, Páez L, Sánchez A, Espinoza F. Bromatología mataratón (Gliricidia sepium) a diferentes edades de corte en Urachiche, estado Yaracuy, Venezuela. Zootecnia Tropical. 2006; 24(4):393-399.

Bhat. (1998). Microbial degradation of tannins a current perspective.

Castellanos AA, Salazar GG, Hernandez MP, Domingez-Araujo G, Barrera CG. 2013. Uso de ensilado de cerdaza en la alimentacion animal. Instituto Nacional de Investigaciones Forestales, Agricolas y Pecuarias. Pp. 2-28.

Castrillón-Quintana O, Jiménez-Pérez RA, Bedoya-Mejía O. Porquinaza en la alimentación animal. Rev Lasallista Investig. 2005; 1(1):72-76.

Cecconello G, Benezra M, Obispo N. Composición química y degradabilidad ruminal de los frutos de algunas especies forrajeras leñosas de un bosque seco tropical. Zootec Trop. 2003;21(2):149-165.

Dominguez G, Arias G, Ruvalcaba G, Hernandez M, Castellanos A, Salazar G. 2013. Efecto de la adición de ensilado de cerdaza en la dieta de vacas en lactación sobre parámetros productivos y las características fisicoquímicas de la leche. Instituto Nacional de Investigaciones Forestales Agrícolas y Pecuarias. Pp. 1-2.

Duarte-Vera F, Magaña-Caballero A, Rodriguez-Garza F. 1990. Utilizacion de heces en la alimentacion animal. Caracterizacion de heces en bovinos y porcinos. Tee Pee Max. 1990;28(1):22-29

Espinal LS. 1968. Visión ecológica del Departamento del Valle del Cauca. Universidad del Valle. Cali. Colombia.

Estrada JÁ, Aranda EMD. 2011. Efecto de la fermentación en estado sólido de la porcinasa sobre la persistencia de patógenos en el ensilaj. Boletin Cientifico Centro de Museo de Historia Natural. Pp.1-10.

Flachowsky G, Ørskov ER. Rumen dry matter degradability of variuos pig faeces and chemically treated pig slurry solids. Arch Anim Nutri. Berlin 1986;36(10):905-913.

Flachowsky G, Baldeweg P, Tiroke K, König H, Astrid Schneider. Feed value and feeding of wastelage made from distillers grain solubles, pig slurry solids and ground straw with urea and $\mathrm{NaOH}$. Biological Wastes. 1990;34(4):271-280. Doi. org/10.1016/0269-7483(90)90029-R

Flachowsky G. Animal Excreta as Feedstuff for RuminantsA Review, J Appl Anim Res. 1997;(12)1:1-40. DOl: 10.1080/09712119.1997.9706185

Galindo BJ, Dominguez AG, Salazar GG, Arteaga CR, Martinez PD, Javier S, Sanchez GG. 2013Ensilado de Cerdaza una Oportunidad para el Manejo de la Bioseguridad y el Microbismo en Granjas Porcinas. Centro de Investigación regional Pacifico Centro.Pp. 1-46.

Garcia-Rodirguez A. 2000. Calidad alimentaria de la mezcla estiercol de cerdo y esquilmos agricolas deshidratada al sol. para bovinos de engorda. Unversidad de Colima. Pp.1-99.

González-García H, Venegas-Maldonado J, Orozco-Erives A, Martínez de la Rosa R, García-San Miguel E, Ramos-Guevara I, Rodríguez-Rivera Á. 2010. La excreta de cerdo como ingrediente alimenticio en la dieta de rumiantes. Ciencia en la frontera: revista de ciencia y tecnología de la UACJ. Pp. 39-45.

Guillermin RJ. 1995. Utilizacion de Cerdaza Fermentadaen al etapa de Destete. Universidad de Guadalajara. Pp. 1-41.
Gupta G, Kelly P. Toxicity (EC 50) comparisons of some animal wastes. Water Air Soil Poll. 1990;53(1-2):113-117.

Hennig AF. 1982. Pig excrement as a new feedstuff for ruminants.

Heredia-Cruz MR, Matamoros I, Zelaya G, Castillo R. 2012. Utilización de cerdaza en dietas de levante para terneros pos destete. Zamorano Escuela Agrícola Panamericana. Departamento de ciencia y producción animal. Pp.3-19.

Hochstrasser, C., Salazar, G., Dominguez, A., Dela-Mora, O., Galindo, B., Xelhuantzi, C., Perez, D. (2012). Tecnologias Alternativas para Contrarestar la Problematica Economica y Social que generan los Residuos Porcicolas. 13 Congreso Nacional de Investigación Sociaeconómica y Ambiental de la Producción Pecuaria, 501-506.

Ondieka JO. The effects of Gliricidia sepium and maize bran as supplementary feed to Rhodes grass hay on intake, digestion and liveweight of dairy goats . Livest Prod Sci. 1998;61:65-67.

Simon BK, Jacobs SWL. Megathyrsus, a new generic name for panicum sub genus. Austrobaileya. 2003;6(3):571-574

Lemos Ramirez JN. 2014. El Matarratón (Gliricidia Sepium) como alternativa para la produccion de leche en ganado bovino. Universidad Nacional Abierta y a Distancia.

Mancini. 1997. Studies on the influence of differently treated molasses/urea mixtures versus soybean meal on parameters of rumen fermentation, duodenal nutrient flow, and in sacco degradation of maize silage and wheat straw in non lactating dairy cows.

Nguyen Van H, Inger L. Performance of growing goats fed Gliricidia maculata . Small Ruminant Res. 2001;39(2):113-119. Doi. org/10.1016/S0921-4488(00)00177-2

Ninabanda-Agualongo JJ. 2012. Alternativasde Manejo de las Excretas Porcinas. Escuela Superior Politecnica de Chimborazo. Pp. 1-75.

Pérez CF, Heredia ER, Flores JS. Engorda de toretes con una dieta integral de excretas frescas de cerdo, melaza y pasto Taiwan. Revista Biomedica. 2002;13:94-99.

Ramírez FJ. 1990. Valor Nutricional de Ensilados de Rastrojo de Maíz y Cerdaza o

Gallinaza para Borregos con o sin implante de Zeranol. Tesis de Maestría. Colegio de Postgraduados. México.

Rojas G, Ojeda Á. Caracterización de los residuos sólidos de efluentes de granjas porcinas y su utilización en vacunos de ceba en confinamiento. Revista Científica, FCV-LUZ, 2002;XII(4):265270.

Sutton AL, Kelly DT, Perry TW. 1988. Performance and carcass characteristics of beef casttle fed diets containing whole corn plant ensiled with swine manure soúds. Journal Paper No. 11,085. Purdue. University Experimental Station.

Granito M, Torres A, Guerra M. Desarrollo y evaluación de una pasta a base de trigo, maíz, yuca y frijol. Interciencia. 2003;28(7):372379.

Trinci APJ, Davies DR, Gull K, Lawrence MI, et al. Anaerobic fungi in herbivorous animals. Mycol Res. 1994;98(2):129-152.

Vollink P. 1993. Comparación de dos dietas con base en forrajes verdes, Gliricidia sepium Vs Tricantera gigantea en el crecimiento de cabretonas. CLEM, Tuluá. 